\title{
The Nano Research Young Innovators (NR45) Awards in nanoenergy
}

\author{
Hailiang Wang ${ }^{1}(\bowtie)$ and Hongjie Dai $^{2}(\bowtie)$ \\ ${ }^{1}$ Department of Chemistry, Yale University, New Haven, CT 06511, USA \\ ${ }^{2}$ Department of Chemistry, Stanford University, Stanford, CA 94305, USA \\ (c) Tsinghua University Press and Springer-Verlag GmbH Germany, part of Springer Nature 2019
}

Following the success of the inaugural 2018 Nano Research Young Innovators (NR45) Awards in nanobiotechnology [1], it is our great pleasure to announce the recipients of the 2019 NR45 Awards in nanoenergy. Congratulations to all the 44 outstanding young investigators under the age of 45 . They were selected through a competitive process by an award committee from the journal's editorial board. Nano Research launched the NR45 Awards program to feature young researchers in various fields of nanoscience and nanotechnology, in recognition of their distinguished accomplishments and/or potential to make substantial contributions to their fields. NR45 awardees are recognized during the Nano Research Symposium integrated in the annual US-SINO Nano Forum, which provides a platform for stimulating communication and fostering collaborations among scientists across the globe. This year, we highlight 44 innovators in the field of energy, covering research foci including batteries, catalysis, fuel cells, photovoltaics, supercapacitors, solar fuels, bioelectricity, and fundamental studies of materials synthesis, structures and properties. Their contributions to this special issue contain 16 review articles and 28 research articles.

This special issue starts with review articles on energy storage materials. Guihua Yu from the University of Texas at Austin reviews the development of stretchable and conductive polymer materials, including molecular design, materials processing and electrode/device engineering, for supercapacitors [2], which are a type of devices that can store and release electrical energy in seconds. Hye Ryung Byon from Korea Advanced Institute of Science and Technology highlights the design principles of organic molecular materials, partially enabled by nanotechnology, for aqueous redox flow batteries [3], a type of devices suitable for grid-scale energy storage. Yuan Yang from Columbia University surveys the importance, preparation and performance of vertically-aligned nanostructures as battery electrodes [4]. Yong-Sheng Hu from Institute of Physics, Chinese Academy of Sciences summarizes the strategies for developing Ni-based layered metal oxide cathode materials for $\mathrm{Na}$ ion batteries [5].

Catalytic materials are another area of focus. Yujie Xiong from University of Science and Technology of China reviews the recent progress in controlling the phases of photocatalyst materials, with new insights into rational phase design for improved performance in energy and environment related reactions [6]. Shuangyin Wang from Hunan University summarizes the design principles and synthetic strategies, including active site exposure, mass transport enhancement, and electron transfer acceleration, for the triple-phase interfaces of electrocatalytic systems involving $\mathrm{H}_{2}, \mathrm{O}_{2}$ and $\mathrm{H}_{2} \mathrm{O}$ [7]. Dingsheng Wang from Tsinghua University reviews the design, synthesis, characterization, and electrocatalytic properties of a type of emerging catalyst materials, i.e. single atom catalysts, derived from metal organic frameworks (MOFs) [8]. Eranda Nikolla from Wayne State University discusses nanoengineering strategies employed to advance electrocatalyst materials for solid oxide fuel cells and electrolyzers [9]. Karthish Manthiram from Massachusetts Institute of Technology provides an in-depth overview of the state-of-the-art heterogenized molecular catalysts for electrochemical $\mathrm{CO}_{2}$ reduction to fuels and useful chemicals [10].

Judy Cha from Yale University summarizes the recent progress on in-situ characterizations of electrochemical intercalation processes for transition metal chalcogenide materials [11]. Yanfeng Zhang from Peking University reviews on-site scanning tunneling microscopy/ spectroscopy investigations of the atomic structures and electronic properties of monolayer $\mathrm{MoS}_{2}$ and its heterostructures, and discusses the implications for electrocatalytic $\mathrm{H}_{2}$ evolution [12]. Hui $\mathrm{Wu}$ from Tsinghua University summaries the synthetic routes for oxygen-deficient metal oxide materials and their applications in catalysis, energy storage, solar cells and pollutant removal [13]. Jing Zhao from University of Connecticut reviews colloidally fabricated metal-quantum emitter nanostructures and discusses the plasmonexciton interactions therein [14]. Chinedum O. Osuji from University of Pennsylvania reviews self-assembly processes of soft matter to form nanostructures and their applications as optical materials and metamaterials [15]. Ardemis A. Boghossian from Ecole Polytechnique Fédérale de Lausanne (EPFL) discusses interfacing biological parts with nanoparticles as an approach to enhance bioelectricity generation in microbial fuel cells and biophotovoltaics [16]. Elad Gross from the Hebrew University of Jerusalem overviews the opportunities and challenges of employing infrared nanospectroscopy to study the chemical information of energy materials [17].

These review articles are followed by original research papers. On battery materials, Yan Yu from University of Science and Technology of China reports $\mathrm{Cu}$-modified multichannel porous $\mathrm{TiO}_{2}$ nanofibers as a high-rate and long-cycle anode material for $\mathrm{Na}$ ion batteries [18]. Shaojun Guo from Peking University presents Co-doped 1T-phase $\mathrm{MoS}_{2}$ nanosheets embedded in N, S co-doped carbon nanobowls showing high rate performance and cycling stability for $\mathrm{Na}$ ion batteries [19]. Qiang Zhang from Tsinghua University reports a sandwich-structured Li metal electrode with improved Li plating and stripping behavior [20], suitable for high-energy rechargeable batteries. Guanglei Cui from Qingdao Institute of Bioenergy and Bioprocess Technology, Chinese Academy of Sciences has developed a flame-retardant quasi-solid polymer electrolyte for rechargeable $\mathrm{Na}$ metal batteries [21]. Ying Shirley Meng from University of California, San Diego reports a polyol method to synthesize layerstructured oxide, spinel-structured oxide and olivine-structured phosphate nanoparticles as cathode materials for Li ion batteries [22].

In the area of electrocatalysis, Yanguang $\mathrm{Li}$ from Soochow 
University introduces a solvent-free nanocasting method to prepare various ordered mesoporous metal sulfide/carbon composite materials with improved electrocatalytic performance for the $\mathrm{H}_{2}$ evolution reaction [23]. Liangbing $\mathrm{Hu}$ from University of Maryland reports a millisecond-scale fast synthesis method to prepare $\mathrm{CoS} / g r a p h e n e$ composite materials for water electrolysis [24]. Jinlong Gong from Tianjin University demonstrates the synthesis of Pt-Pd bilayer nanocages improving the catalytic activity for $\mathrm{H}_{2}$ evolution [25]. Karl J. J. Mayrhofer from Max-Planck-Institut für Eisenforschung $\mathrm{GmbH}$ describes a liquid atomic layer deposition method toward maximized utilization of iridium for catalyzing electrochemical water oxidation to $\mathrm{O}_{2}$ in acidic solutions [26]. Zhangquan Peng from Changchun Institute of Applied Chemistry, Chinese Academy of Sciences reports a mesoporous ternary Co-Fe-Ni oxide material with a hierarchical bimodal channel structure as both the anode catalyst and the cathode catalyst for water electrolysis [27]. Shannon W. Boettcher from University of Oregon reports composition-dependent electronic structures and catalytic activities of ternary $\mathrm{Ni}-\mathrm{Co}-\mathrm{Fe}$ oxyhydroxide materials as electrocatalysts for the $\mathrm{O}_{2}$ evolution reaction [28]. Changzheng Wu from University of Science and Technology of China reports the development of an active $\mathrm{O}_{2}$ evolution electrocatalyst based on an oxygen-deficient perovskite-structured Sr-Co oxide [29]. Xiaoming Sun from Beijing University of Chemical Technology has invented a new approach of using cationic surfactants to construct superaerophobic nanoarray electrodes with enhanced catalytic performance for water oxidation [30]. Jin Suntivich from Cornell University presents the substitutional effects of $3 \mathrm{~d}$ transition metals in molybdenum/tungsten nitride for electrocatalytic $\mathrm{O}_{2}$ reduction in acid [31].

Dehui Deng from Dalian Institute of Chemical Physics, Chinese Academy of Sciences describes single $\mathrm{Fe}$ atoms confined in a multiscale carbon foam exhibiting high activity for electrocatalytic reduction of $\mathrm{CO}_{2}$ to $\mathrm{CO}$ [32]. Xinbo Zhang from Changchun Institute of Applied Chemistry, Chinese Academy of Sciences reports an Fe-N-C electrocatalyst material that can convert $\mathrm{CO}_{2}$ and $\mathrm{H}_{2} \mathrm{O}$ to $\mathrm{CO} / \mathrm{H}_{2}$ mixtures of different ratios [33]. Gengfeng Zheng from Fudan University reports that increased $\mathrm{N}$ doping in MOF-derived mesoporous carbon leads to enhanced electrocatalytic activity for $\mathrm{CO}_{2}$ reduction to $\mathrm{CO}$ [34]. Yongye Liang from Southern University of Science and Technology emphasizes the importance of dispersing metal phthalocyanine molecules on the surface of highly-conductive carbon nanotubes to exposing the intrinsic electrocatalytic properties of these molecular materials for $\mathrm{CO}_{2}$ reduction reactions [35].

Thermal catalysis and relevant materials are also covered. Xiaoqing Huang from Soochow University reports the tuning of surface electronic structure of $\mathrm{Pt}-\mathrm{Pb}$ bimetallic nanocrystals to enhance their catalytic performance for the ethanol reforming reaction to produce clean $\mathrm{H}_{2}$ fuel [36]. Ding Ma from Peking University has developed a MOF-derived Fe-based catalyst for efficient conversion of $\mathrm{N}_{2}$ and $\mathrm{H}_{2}$ to ammonia [37]. Qiang Fu from Dalian Institute of Chemical Physics, Chinese Academy of Sciences reports controlled growth of uniform monolayer and bilayer $\mathrm{ZnO}$ nanostructures on $\mathrm{Au}(111)$ surface and their hydroxylation under catalysis-relevant conditions [38]. Jovana Zečević from Utrecht University has studied the structural stability of a series of oxide nanoparticles using liquid phase transmission electron microscopy and demonstrates a correlation between stability and Gibbs free energy of hydration [39]. Aleksandra Vojvodic from University of Pennsylvania presents a theory and experiment combined study of the structure and distribution of Fe dopants in two-dimensional (2D) CoO nanoislands on $\mathrm{Au}(111)$ as a function of the Fe/Co ratio [40]. Kristie J. Koski from University of California, Davis reports pressure-induced semiconductor-to-metal phase transition behavior for 2D layered silicon telluride nanoplates [41].

On solar energy conversion, Dunwei Wang from Boston College reports the influences of Pt catalyst loading on the charge transfer and recombination processes at the interface of a GaN-protected Si photoanode [42]. Tierui Zhang from Technical Institute of Physics and Chemistry, Chinese Academy of Sciences has developed a polymer-templated method to synthesize holey graphitic carbon nitride nanotubes with enhanced photocatalytic activity for $\mathrm{H}_{2}$ production under visible-light irradiation [43]. Robert Baker from the Ohio State University reports on the influences of $\mathrm{Cu}$ vacancies, interstitial $\mathrm{O}$ atoms, and phase impurities on the electron dynamics and photochemistry of $\mathrm{Cu}-\mathrm{Fe}$ oxide photocathodes [44]. Wei You from University of North Carolina at Chapel Hill reports their discovery that more fluorination on both the donor polymer and the non-fullerene acceptor leads to higher overall efficiency for the solar cell [45].

Overall, this issue highlights a diverse range of topics on broadlydefined nanomaterials for energy conversion and storage. We sincerely thank all the awardees for their excellent contributions to this 2019 NR45 Special Issue. We hope that you will enjoy this special issue, and encourage you and your colleagues to nominate outstanding young innovators in your field for the following years' NR45 awards.

\section{References}

[1] Gu, Z.; Dai, H. J. The inaugural Nano Research Young Innovators (NR45) Award in nanobiotechnology. Nano Res. 2018, 11, 4931-4935.

[2] Wang, Y. Q.; Ding, Y.; Guo, X. L.; Yu, G. H. Conductive polymers for stretchable supercapacitors. Nano Res. 2019, 12, 1978-1987.

[3] Singh, V.; Kim, S.; Kang, J.; Byon, H. R. Aqueous organic redox flow batteries. Nano Res. 2019, 12, 1988-2001.

[4] Wang, X.; Wang, T. Y.; Borovilas, J.; He, X. D.; Du, S. Y.; Yang, Y. Verticallyaligned nanostructures for electrochemical energy storage. Nano Res. 2019, 12, 2002-2017.

[5] Zhao, C. L.; Lu, Y. X.; Chen, L. Q.; Hu, Y.-S. Ni-based cathode materials for Na-ion batteries. Nano Res. 2019, 12, 2018-2030.

[6] Bai, S.; Gao, C.; Low, J. X.; Xiong, Y. J. Crystal phase engineering on photocatalytic materials for energy and environmental applications. Nano Res. 2019, 12, 2031-2054.

[7] Wang, Y. Q.; Zou, Y. Q.; Tao, L.; Wang, Y. Y.; Huang, G.; Du, S. Q.; Wang, S. Y. Rational design of three-phase interfaces for electrocatalysis. Nano Res. 2019, 12, 2055-2066.

[8] Sun, T. T.; Xu, L. B.; Wang, D. S.; Li, Y. D. Metal organic frameworks derived single atom catalysts for electrocatalytic energy conversion. Nano Res. 2019, 12, 2067-2080.

[9] Carneiro, J.; Nikolla, E. Nanoengineering of solid oxide electrochemical cell technologies: An outlook. Nano Res. 2019, 12, 2081-2092.

[10] Corbin, N.; Zeng, J.; Williams, K.; Manthiram, K. Heterogeneous molecular catalysts for electrocatalytic $\mathrm{CO}_{2}$ reduction. Nano Res. 2019, 12, 2093-2125.

[11] Yazdani, S.; Yarali, M.; Cha, J. J. Recent progress on in situ characterizations of electrochemically intercalated transition metal dichalcogenides. Nano Res. 2019, 12, 2126-2139.

[12] Hong, M.; Shi, J. P.; Huan, Y. H.; Xie, Q.; Zhang, Y. F. Microscopic insights into the catalytic mechanisms of monolayer $\mathrm{MoS}_{2}$ and its heterostructures in hydrogen evolution reaction. Nano Res. 2019, 12, 2140-2149.

[13] Zu, D.; Wang, H. Y.; Lin, S.; Ou, G.; Wei, H. H.; Sun, S. Q.; Wu, H. Oxygendeficient metal oxides: Synthesis routes and applications in energy and environment. Nano Res. 2019, 12, 2150-2163.

[14] Luo, Y.; Zhao, J. Plasmon-exciton interaction in colloidally fabricated metal nanoparticle-quantum emitter nanostructures. Nano Res. 2019, 12, 2164-2171.

[15] Gabinet, U. R.; Osuji, C. O. Optical materials and metamaterials from nanostructured soft matter. Nano Res. 2019, 12, 2172-2183.

[16] Mouhib, M.; Antonucci, A.; Reggente, M.; Amirjani, A.; Gillen, A. J.; Boghossian, A. A. Enhancing bioelectricity generation in microbial fuel cells and biophotovoltaics using nanomaterials. Nano Res. 2019, 12, 2184-2199.

[17] Gross, E. Challenges and opportunities in IR nanospectroscopy measurements of energy materials. Nano Res. 2019, 12, 2200-2210.

[18] Wu, Y.; Wei, Z. X.; Xu, R.; Gong, Y.; Gu, L.; Ma, J. M.; Yu, Y. Boosting the rate capability of multichannel porous $\mathrm{TiO}_{2}$ nanofibers with well-dispersed 
$\mathrm{Cu}$ nanodots and $\mathrm{Cu}^{2+}$-doping derived oxygen vacancies for sodium-ion batteries. Nano Res. 2019, 12, 2211-2217

[19] Li, P. H.; Yang, Y.; Gong, S.; Lv, F.; Wang, W.; Li, Y. J.; Luo, M. C.; Xing, Y.; Wang, Q; Guo, S. J. Co-doped $1 \mathrm{~T}-\mathrm{MoS}_{2}$ nanosheets embedded in N, S-doped carbon nanobowls for high-rate and ultra-stable sodium-ion batteries. Nano Res. 2019, 12, 2218-2223.

[20] Li, T.; Shi, P.; Zhang, R.; Liu, H.; Cheng, X.-B.; Zhang, Q. Dendrite-free sandwiched ultrathin lithium metal anode with even lithium plating and stripping behavior. Nano Res. 2019, 12, 2224-2229.

[21] Yang, J. F.; Zhang, M.; Chen, Z.; Du, X. F.; Huang, S. Q.; Tang, B.; Dong, T. T.; Wu, H.; Yu, Z.; Zhang, J. J. et al. Flame-retardant quasi-solid polymer electrolyte enabling sodium metal batteries with highly safe characteristic and superior cycling stability. Nano Res. 2019, 12, 2230-2237.

[22] Chung, H.; Grenier, A.; Huang, R.; Wang, X. F.; Lebens-Higgins, Z.; Doux, J.-M.; Sallis, S.; Song, C. Y.; Ercius, P.; Chapman, K. et al. Comprehensive study of a versatile polyol synthesis approach for cathode materials for Li-ion batteries. Nano Res. 2019, 12, 2238-2249.

[23] Zhu, J. H.; Chen, Z.; Jia, L.; Lu, Y. Q.; Wei, X. R.; Wang, X. N.; Wu, W. D.; Han, N.; Li, Y. G.; Wu, Z. X. Solvent-free nanocasting toward universal synthesis of ordered mesoporous transition metal sulfide@N-doped carbon composites for electrochemical applications. Nano Res. 2019, 12, 2250-2258.

[24] Chen, Y. N.; Xu, S. M.; Zhu, S. Z.; Jacob, R. J.; Pastel, G.; Wang, Y. B.; Li, Y. J.; Dai, J. Q.; Chen, F. J.; Xie, H. et al. Millisecond synthesis of CoS nanoparticles for highly efficient overall water splitting. Nano Res. 2019, 12, 2259-2267.

[25] Wang, Y. H.; Zhang, L.; Hu, C. L.; Yu, S. N.; Yang, P. P.; Cheng, D. F.; Zhao, Z.-J.; Gong, J. L. Fabrication of bilayer Pd-Pt nanocages with sub-nanometer thin shells for enhanced hydrogen evolution reaction. Nano Res. 2019, 12, 2268-2274.

[26] Ledendecker, M.; Geiger, S.; Hengge, K.; Lim, J.; Cherevko, S.; Mingers, A. M.; Göhl, D.; Fortunato, G. V.; Jalalpoor, D.; Schüth, F. et al. Towards maximized utilization of iridium for the acidic oxygen evolution reaction. Nano Res. 2019, 12, 2275-2280.

[27] Han, L. L.; Guo, L. M.; Dong, C. Q.; Zhang, C.; Gao, H.; Niu, J. Z.; Peng, Z. Q.; Zhang, Z. H. Ternary mesoporous cobalt-iron-nickel oxide efficiently catalyzing oxygen/hydrogen evolution reactions and overall water splitting. Nano Res. 2019, 12, 2281-2287.

[28] Stevens, M. B.; Enman, L. J.; Korkus, E. H.; Zaffran, J.; Trang, C. D. M.; Asbury, J.; Kast, M. G.; Toroker, M. C.; Boettcher, S. W. Ternary Ni-Co-Fe oxyhydroxide oxygen evolution catalysts: Intrinsic activity trends, electrical conductivity, and electronic band structure. Nano Res. 2019, 12, 2288-2295.

[29] Wu, J. C.; Guo, Y. Q.; Liu, H. F.; Zhao, J. Y.; Zhou, H. D.; Chu, W. S.; Wu, C. Z. Room-temperature ligancy engineering of perovskite electrocatalyst for enhanced electrochemical water oxidation. Nano Res. 2019, 12, 2296-2301

[30] Xie, Q. X.; Zhou, D. J.; Li, P. S.; Cai, Z.; Xie, T. H.; Gao, T. F.; Chen, R. D.; Kuang, Y.; Sun, X. M. Enhancing oxygen evolution reaction by cationic surfactants. Nano Res. 2019, 12, 2302-2306.

[31] Fritz, K. E.; Yan, Y. C.; Suntivich, J. Influence of 3d transition-metal substitution on the oxygen reduction reaction electrocatalysis of ternary nitrides in acid. Nano Res. 2019, 12, 2307-2312.

[32] Zhang, Z.; Ma, C.; Tu, Y. C.; Si, R.; Wei, J.; Zhang, S. H.; Wang, Z.; Li, J.-F.;
Wang, Y.; Deng, D. H. Multiscale carbon foam confining single iron atoms for efficient electrocatalytic $\mathrm{CO}_{2}$ reduction to CO. Nano Res. 2019, 12, 2313-2317.

[33] Zhong, H. X.; Meng, F. L.; Zhang, Q.; Liu, K. H.; Zhang, X. B. Highly efficient and selective $\mathrm{CO}_{2}$ electro-reduction with atomic Fe-C-N hybrid coordination on porous carbon nematosphere. Nano Res. 2019, 12, 2318-2323.

[34] Kuang, M.; Guan, A. X.; Gu, Z. X.; Han, P.; Qian, L. P.; Zheng, G. F. Enhanced N-doping in mesoporous carbon for efficient electrocatalytic $\mathrm{CO}_{2}$ conversion. Nano Res. 2019, 12, 2324-2329.

[35] Jiang, Z.; Wang, Y.; Zhang, X.; Zheng, H. Z.; Wang, X. J.; Liang, Y. Y. Revealing the hidden performance of metal phthalocyanines for $\mathrm{CO}_{2}$ reduction electrocatalysis by hybridization with carbon nanotubes. Nano Res. 2019, 12, 2330-2334.

[36] E, B.; Huang, B. L.; Zhang, N.; Shao, Q.; Li, Y. J.; Huang, X. Q. Enhancing catalytic $\mathrm{H}_{2}$ generation by surface electronic tuning of systematically controlled Pt-Pb nanocrystals. Nano Res. 2019, 12, 2335-2340.

[37] Yan, P. Q.; Guo, W. H.; Liang, Z. B.; Meng, W.; Yin, Z.; Li, S. W.; Li, M. Z.; Zhang, M. T.; Yan, J.; Xiao, D. Q. et al. Highly efficient K-Fe/C catalysts derived from metal-organic frameworks towards ammonia synthesis. Nano Res. 2019, 12, 2341-2347.

[38] Wu, H.; Fu, Q.; Li, Y. F.; Cui, Y.; Wang, R.; Su, N.; Lin, L.; Dong, A. Y.; Ning, Y. X.; Yang, F.; Bao, X. H. Controlled growth of uniform two-dimensional $\mathrm{ZnO}$ overlayers on $\mathrm{Au}(111)$ and surface hydroxylation. Nano Res. 2019, 12, 2348-2354.

[39] Meijerink, M. J.; de Jong, K. P.; Zečević, J. Assessment of oxide nanoparticle stability in liquid phase transmission electron microscopy. Nano Res. 2019, 12, 2355-2363.

[40] Curto, A.; Sun, Z. Z.; Rodríguez-Fernández, J.; Zhang, L.; Parikh, A.; Tan, T.; Lauritsen, J. V.; Vojvodic, A. Anisotropic iron-doping patterns in two-dimensional cobalt oxide nanoislands on $\mathrm{Au}(111)$. Nano Res. 2019, $12,2364-2372$.

[41] Johnson, V. L.; Anilao, A.; Koski, K. J. Pressure-dependent phase transition of 2D layered silicon telluride $\left(\mathrm{Si}_{2} \mathrm{Te}_{3}\right)$ and manganese intercalated silicon telluride. Nano Res. 2019, 12, 2373-2377.

[42] He, Y. M.; Vanka, S.; Gao, T. Y.; He, D.; Espano, J.; Zhao, Y. Y.; Dong, Q.; Lang, C. C.; Wang, Y. J.; Hamann, T. W.; Mi, Z. T.; Wang, D. W. Dependence of interface energetics and kinetics on catalyst loading in a photoelectrochemical system. Nano Res. 2019, 12, 2378-2384.

[43] Wang, X. S.; Zhou, C.; Shi, R.; Liu, Q. Q.; Waterhouse, G. I. N.; Wu, L. Z.; Tung, C.-H.; Zhang, T. R. Supramolecular precursor strategy for the synthesis of holey graphitic carbon nitride nanotubes with enhanced photocatalytic hydrogen evolution performance. Nano Res. 2019, 12, 2385-2389.

[44] Fugate, E. A.; Biswas, S.; Clement, M. C.; Kim, M.; Kim, D.; Asthagiri, A; Baker, L. R. The role of phase impurities and lattice defects on the electron dynamics and photochemistry of $\mathrm{CuFeO}_{2}$ solar photocathodes. Nano Res. 2019, 12, 2390-2399.

[45] Bauer, N.; Zhang, Q. Q.; Rech, J. J.; Dai, S. X.; Peng, Z. X.; Ade, H.; Wang, J. Y.; Zhan, X. W.; You, W. The impact of fluorination on both donor polymer and non-fullerene acceptor: The more fluorine, the merrier. Nano Res. 2019, 12, 2400-2405. 